\title{
Duration of immunity of a four-valent vaccine against bovine respiratory diseases
}

\author{
Corinne Philippe-Reversat ${ }^{1}$, David Homer ${ }^{1}$, Claude Hamers ${ }^{1}$, Sylvie Brunet ${ }^{1}$, \\ Milan Huňady² \\ ${ }^{1}$ Merial, Lyon, France \\ ${ }^{2}$ Bioveta a.s., Ivanovice na Hané, Czech Republic \\ Received February 22, 2017 \\ Accepted December 19, 2017
}

\begin{abstract}
This study demonstrated the duration of immunity over 6 months of a vaccine against key bovine respiratory disease pathogens: Parainfluenza 3, Bovine Respiratory Syncytial Virus, Bovine Viral Diarrhoea and Mannheimia haemolytica. This was performed by challenge on colostrumdeprived calves at the age of 2 weeks. Recent European field isolates were used as challenge strains. Clinical signs and pathogen excretion or presence were monitored. Field relevance of the viral challenge strains was analysed using phylogenic analysis. Significant reduction of excretion of the 3 viruses in vaccinated animals was a consistent finding, demonstrating the efficacy of the vaccine. Reducing shedding is indeed key to interrupting the infection transmission chain and helping to achieve the protective effects of immunisation that extend beyond the individual. A significant reduction of clinical signs and lung lesions following the Mannheimia haemolytica challenge was also observed in vaccinated animals versus controls. Comparison of the challenge strains to an array of global and European strains, including recent ones, demonstrated a high genetic proximity, supporting the potential for the vaccine to maintain similar levels of efficacy in the field over a 6-month period post vaccination.
\end{abstract}

Cattle, immunization, Parainfluenza 3, Bovine Respiratory Syncytial Virus, Bovine Viral Diarrhoea, Mannheimia haemolytica

Bovine Respiratory Disease (BRD) is a multifactorial disease impacting cattle production. The disease is often referred to as a disease complex, involving both bacterial and viral pathogens as well as psychological (e.g. stress) and physical (e.g. air quality and temperature) factors in various combinations. It is recognized as having a major impact on the health and economics of beef and dairy production and is considered as the most important illness associated with feedlot cattle in the USA (Snowder et al. 2006) and possibly worldwide (Horwood et al. 2008). Bovine Respiratory Disease is a relatively common phenomenon in younger cattle, especially those housed in intensive conditions, with vaccination programmes against BRD commencing on some farms in calves as young as 2 weeks of age.

The importance of the disease in young calves and growing animals requires the vaccines and programmes used from the outset to be as effective and appropriate as possible. The vaccine antigens being used need to be appropriate for the strains currently (and at risk of) circulating on farms. They also need to be presented efficiently to the vaccinated animal so as to ensure both an optimal primary vaccination course response and a long duration of protective immunity. The immuno-competence of vaccinated calves and the level of circulating maternally-derived colostral antibodies (MDA) present can also have an impact on the vaccination response. These factors and the specifics of the disease dynamics on farm are all important when determining the right vaccination approach.

The study described herein provides an initial assessment of the relevance of the Bovalto Respi range of respiratory vaccines for the vaccination of young calves against four majors

Address for correspondence:

Corinne Philippe-Reversat

Merial

29 avenue Tony Garnier

Phone: $+33(0) 472720000$

69007 Lyon, France 
BRD pathogens: Parainfluenza 3 (Pi3), Bovine Respiratory Syncytial Virus (BRSV), Bovine Viral Diarrhoea (BVD) and Mannheimia haemolytica (Mh). As per regulatory requirements, colostrum deprived calves were used, hence the study did not consider the relevance of and approaches for MDAs. This study confirms the efficacy of the antigens (type and presentation), the likelihood of significant duration of immunity being achievable from the vaccine ( 6 months) and discusses the relevance of the antigens used to current circulating pathogens in Europe.

Four different vaccination-challenge studies were carried out to investigate the duration of immunity provided by Bovalto Respi $4^{\circledR}$ against each of the strains included in the vaccine, i.e. Pi3, BRSV, BVD, and Mannheimia haemolytica. The design was similar for each study, apart from the challenge materials themselves and the type of follow-up that was used (adapted to each pathogen).

\section{Vaccine efficacy}

\section{Materials and Methods}

Efficacy requirements of the immunogenicity test of the corresponding European Pharmacopoeia (Ph. Eur.) monographs were applied when existing (or by default, monographs dealing with similar antigen were used). The consent of the Ethics Committee on the use of animals for the trial was obtained.

Two-week-old colostrum deprived calves were vaccinated twice at 21-day intervals and challenged 6 months after the $2^{\text {nd }}$ injection. Ten calves were used for the viral studies and divided into two groups, one of 5 vaccinated and one of 5 controls. Sixteen calves were used for Mannheimia haemolytica with groups of 8 each for the vaccinated animals and controls.

Animals were observed daily during the 14 days after challenge for the viral strains in order to detect clinical symptoms of respiratory disease such as sneezing, nasal discharge, coughing, etc. A scoring table was used for each of the criteria. Rectal temperature was also recorded daily and temperatures above $40{ }^{\circ} \mathrm{C}$ were considered as hyperthermia. Nasal swabs were systematically performed, starting on the day of challenge and subsequently daily from the $3^{\text {rd }}$ day post-challenge until the end of the study.

The follow-up of calves challenged by Mannheimia haemolytica (M. haemolytica) was shorter, lasting just 7 days (as per Ph. Eur. 1944 recommendations for the immunogenicity test). Daily clinical observations for respiratory signs as well as rectal temperature were recorded. A post mortem examination was performed to identify lung lesions caused by $M$. haemolytica and to re-isolate the bacteria. Indeed, $M$. haemolytica is commensal in the upper respiratory tract, becoming pathogenic once it has reached the lungs where it causes severe pathology to lung tissue such as fibrinous changes and necrosis.

Table 2 below recapitulates details on the challenge materials used in the vaccine efficacy studies (virus details and inoculation approach).

Serology was performed on blood samples to determine the level of antibodies for the viral strains using virus neutralisation technique and results were expressed as $\mathrm{PD}_{50}$ (serum dilution protecting $50 \%$ of the infected wells). A classical enzyme-linked immunosorbent assay (ELISA) was performed to assess the level of $M$. haemolytica antibodies.

Nasal swabs were immediately diluted and inoculated into MDBK cell line suspension on 96-well plates. After incubation for 5 days, Pi3 titre was determined based on its specific cytopathogenic effect whilst immunofluorescence staining was used to reveal and titrate the levels of BVD and BRSV. Results were expressed in $\mathrm{TCID}_{50}(50 \%$ Tissue Culture Infective Dose).

In the course of $M$. haemolytica challenge, post mortem lungs samples were cultivated in a liquid medium in tubes. The positivity of the samples for M. haemolytica was then assessed.

The level of difference between vaccinated and control groups was tested by comparison of two independent selections using the QC Expert 3.1 program and the significance level $\alpha=0.05$. First, the compliance of variances of both selections was tested by the Fisher-Snedecor test and based on the test results a matching $t$-test (for the same or different variances) was subsequently used for testing of the compliance of the mean values.

\section{Phylogenic analysis}

The challenge strains identified in Table 2 were all heterologous from the vaccine strains used in the range of Bovalto Respi vaccines and were all isolated between 2003-2006 from infected calves.

Repeated challenges to account for all strains in the field cannot be justified for obvious ethical reasons so it is indeed critical not only to obtain significant clinical results after the challenge but also to ensure that the strains used in challenges are as representative as possible of those likely to be met in the field.

A phylogenic analysis was then performed, targeting genes chosen both for being important for antigenic variation in the field and also for the development of protection. These major viral antigens on the viral challenge strains were compared with published sequences (GeneBank) of field strains by Clustal alignment (Seaview software) and phylogenic tree (distance method Neibourg-Joining, Kimura correction, 500 bootstrap replicates; only bootstrap values superior to 70 were considered as significant). The percentages of homology were deduced from alignment. 
Table 1. High level design of the trials.

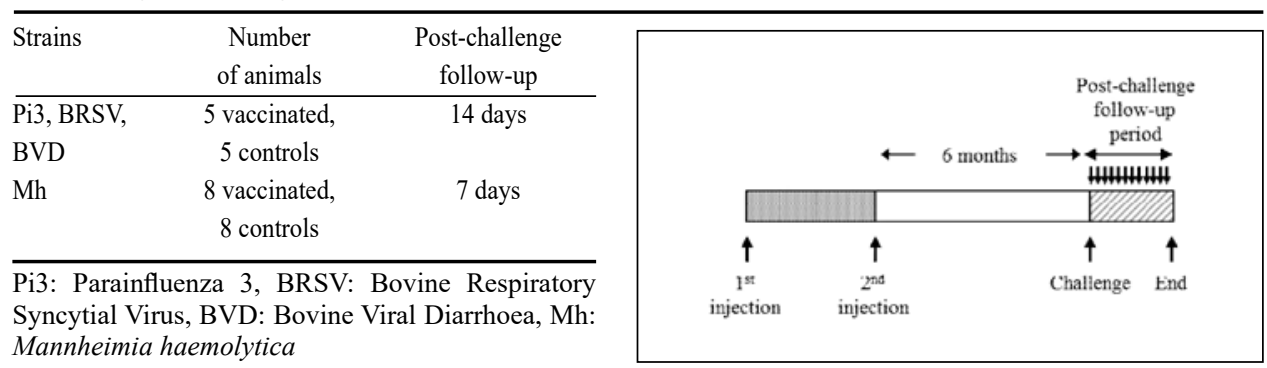

Table 2. Origin, dose and route of administration of the challenge materials.

\begin{tabular}{llcll}
\hline Strains & \multicolumn{1}{c}{ Challenge strains origin } & $\begin{array}{c}\text { Date } \\
\text { of isolation }\end{array}$ & Titre & Dose and route of administration \\
\hline Pi3 & Field isolates acquired from & $2003^{*}$ & $10^{7} \mathrm{TCID}_{50} / \mathrm{ml}$ & $5 \mathrm{ml}$ sprayed into nostrils \\
BRSV & the Veterinary Research Institute & $2003^{*}$ & $10^{5,3} \mathrm{TCID}_{50} / \mathrm{ml}$ & $5 \mathrm{ml}$ sprayed into nostrils \\
BVD & in Brno & $2005^{*}$ & $10^{6} \mathrm{TCID}_{50} / \mathrm{ml}$ & $5 \mathrm{ml}$ sprayed into nostrils \\
& Field isolate of Mannheimia & & & \\
Mh & haemolytica A1 from State & $2006^{* *}$ & $5 \times 10^{9} \mathrm{CFU} / \mathrm{ml}$ & $5 \mathrm{ml}$ by intra-tracheal administration \\
& Vet. Inst., Olomouc, CZ & & & \\
\hline
\end{tabular}

* from a clinically diseased calf nasal swab ** from a clinically diseased calf lung

$\mathrm{TCID}_{50}: 50 \%$ Tissue culture Infective Dose, CFU: colony-forming unit, Pi3: Parainfluenza 3, BRSV: Bovine Respiratory Syncytial Virus, BVD: Bovine Virus Diarrhoea, Mh: Mannheimia haemolytica

\section{Vaccine efficacy}

\section{Results}

Very few and mild clinical signs were observed during the viral challenges. However, a consistent excretion of the virus was detected as shown in Fig. 1 describing the evolution of the mean titre of virus in nasal swabs in vaccinated and control calves following the challenge 6 months after vaccination.

All vaccinated animals showed a significant reduction on both the titre of excreted virus and the duration of virus excretion compared to the non-vaccinated group $(P=0.02$ for the viral load and $P<0.001$ for the duration for Pi3; $P<0.001$ for both viral load and duration of excretion for BRSV and BVD). The results are shown in Fig. 1.

For M. haemolytica, a significant reduction of clinical signs was demonstrated $(P=0.01)$. Indeed, whereas none of the vaccinated animals were affected, up to 5 controls out of 8 showed one or more clinical signs such as apathy, coughing, dyspnoea and nasal discharge for at least 3 days. The results are shown in Fig. 2.

In addition, the lung lesion scores reflected in the graphs were also significantly different, in line with the clinical observations $(P=0.001)$. Lung scores were calculated according to the degree of lung affection using a scale from 0 (no pathology found) to 20 (global affection with pleuritic) for each individual calf. The results are shown in Fig. 3.

\section{Phylogenic analysis}

Phylogenic analysis of the Pi3 challenge strain confirmed it was representative of circulating $\mathrm{Pi} 3$ strains. Indeed, on the target hemagglutinin-neuraminidase $(\mathrm{HN})$ protein gene compared with 17 published sequences of a worldwide origin, the challenge strain demonstrated $94 \%$ homology. Data also confirmed that it was a classical genotype A Pi3 strain. 

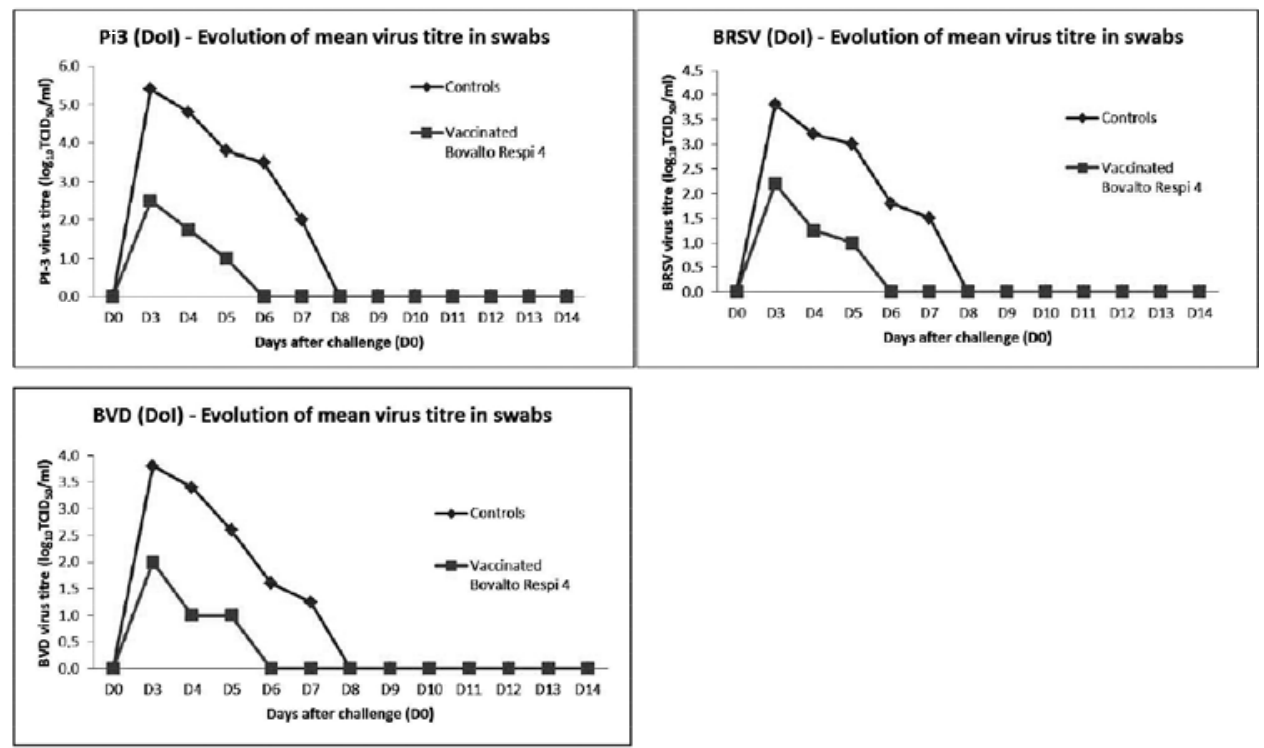

Pi3: Parainfluenza 3, BRSV: Bovine Respiratory Syncytial Virus, BVD: Bovine Viral Diarrhoea, DOI: Duration of immunity, $\mathrm{TCID}_{50}: 50 \%$ Tissue Culture Infective Dose

Fig. 1. Evolution of mean titres in swabs post-challenge for Pi3, BRSV and BDV strains

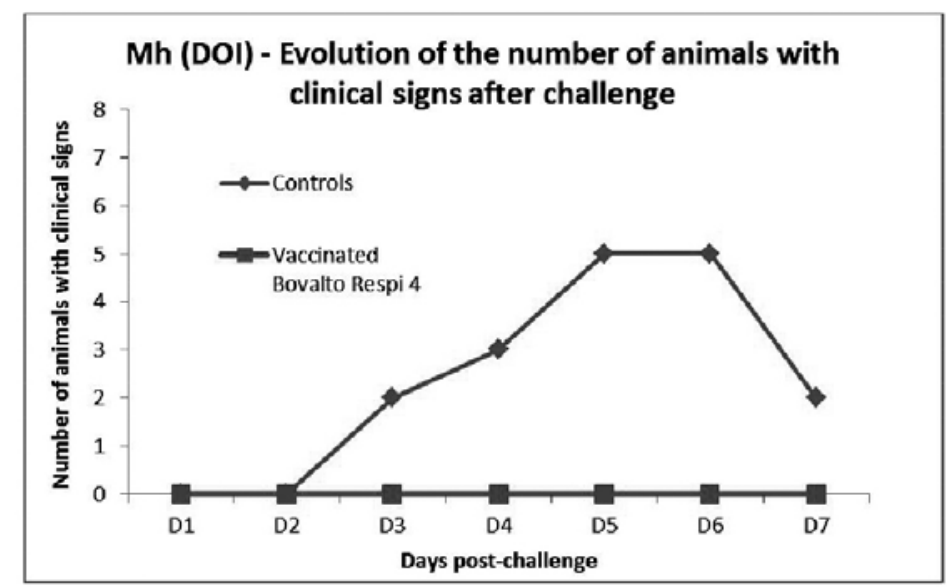

Mh: Mannheimia haemolytica, DOI: duration of immunity

Fig. 2. Evolution of the number of animals with clinical signs after the Mannheimia haemolytica challenge

For BRSV, the phylogenic tree determination identified that the challenge strain belonged to group $\mathrm{AB}$, i.e. intermediary antigen group, along with some Danish, Belgian and Dutch isolates. Phylogenic analysis on the target gene $\mathrm{G}$ against 31 published sequences determined a minimum of $93.5 \%$ homology. 


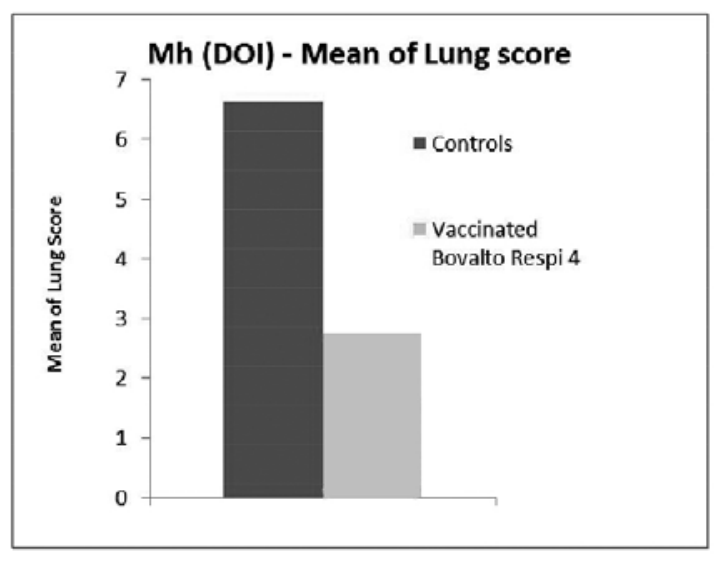

Mh: Mannheimia haemolytica, DOI: duration of immunity

Fig. 3. Comparison of lung scores after the Mannheimia haemolytica challenge in vaccinated animals and controls
The phylogenic analysis on the BVD challenge strain targeted the E2 gene, comparing it with 70 published sequences. The analysis demonstrated $87 \%$ homology. Furthermore, the analysis confirmed that the challenge strain belongs to the BVDV genotype 1, which is the most prevalent in the field in Europe (and worldwide).

The relevance of the $M$. haemolytica vaccine strain was not assessed by phylogenic analysis. However, the challenge strain used was Mh A1 which prevails all over the world and is the major causative agent of bovine mannheimiosis (Zechinon et al. 2005) or bovine pneumonia (Rice et al. 2007), also in Europe (Ewers et al. 2004). It is the most important pathogenic strain in the field, with $70 \%$ of serotyped isolates being A1 as shown in various surveys in Germany and the USA (Ames 2007).

\section{Discussion}

In most of the laboratory studies presented here, only low to mild clinical signs were observed in both vaccinated and control animals, often not enabling a statistical differentiation to be determined between the vaccinated animals and controls with regards to this criteria. This is not surprising as BRD is a multifactorial disease (Valarcher and Hägglund 2006) and results from the combination of several etiological agents which increase the severity of the infection, furthermore worsened by environmental factors.

For example, Pi3 is often considered as a facilitator rather than a primary pathogen. It is described as having a role in enabling subsequent bacterial infection such as $M$. haemolytica. Similarly persistent infection (PI) with bovine virus diarrhoea virus, can increase BRD occurrence in calves (Bednarek et al. 2012). These field conditions cannot be fully reproduced in controlled laboratory conditions where only one unique pathogen is inoculated to animals maintained in a proper and controlled environment (as per regulatory requirements). Hence the low level of clinical signs observed, except in $M$. haemolytica challenges, were they are to be expected.

Nevertheless, virus excretion was a consistent finding after respiratory challenges and this excretion is a reliable marker for demonstration of vaccine efficacy. Indeed, the pathogen load dispatched in the environment is directly linked to the development of BRD in the herd (Callan 2002; Valarcher and Hägglund 2006). Minimising pathogen shedding is key to interrupting the infection transmission chain, enabling the protective effects of immunisation to be extended beyond the individual.

The observed clinical protection as well as the significant reduction of the bacterial invasion in lungs against the $M$. haemolytica challenge is of utmost importance given the severity of the associated pneumonia that can develop, which is often fatal to the calves.

These data support the long-lasting and constant efficacy of Bovalto Respi $4^{\circledR}$ vaccine over a 6-month period, confirmed by the challenge against four of the key viral and bacterial pathogens implicated in BRD. The phylogenic analysis also showed sufficient 
genetic proximity between the challenge viruses from these studies and numerous known and published field strains assessed to conclude that the results can be extrapolated to the field. This confirms the relevance of the challenge strains and further supports the extrapolation of these efficacy results to the field. This is an important observation as there are reports for at least some of the viruses of a level of antigenic diversity in the field that could potentially affect the ability of vaccines to provide protection.

$\mathrm{Pi} 3$ is associated with some structural variation of its envelope but this diversity seems to be more associated with variations in pathogenicity than with a potential for reduced vaccine efficacy (Ellis 2010). In general terms there is a high level of genomic conservation among bovine Pi3 isolates tested (Ellis 2010). One study demonstrated greater than $92 \%$ nucleotide identity across the entire genome, reflecting a similar level of conservation with bovine Pi3 to that displayed between Human Parainfluenza Virus type 3 (HPIV-3) isolates (Horw ood et al. 2008). However, Henrickson (2003) suggested a slow progressive antigenic change for HPIV that will cause older standard reference sera to not universally react in routine serologic assays so there is some potential for antigenic diversity in the family.

The key antigens to consider for $\mathrm{Pi} 3$ are those associated with neuraminadase (NA) activity and the F-protein. These function to enable penetration of the mucus layer as well as the binding to and entry into cells. Strains with strong NA activity can more effectively infect and affect younger calves (Ellis 2010). Neuraminadase is a part of the HN protein and our phylogenic analysis confirmed high homology between the challenge strain used and the published field strains for this key protein.

For BRSV, four antigenic subgroups (A, B, AB, and untyped) have been established, with the subgroup B not being detected since 1976 (Valarcher and Taylor 2007). The extent of antigenic variation observed among BRSV isolates is, however, considerably lesser than that observed with Human Respiratory Syncytial Virus (HRSV) isolates (Meyer et al. 2008). The degree of genetic variability of the BRSV genome is indeed described as limited, being less than 15\% (Valarcher and Taylor 2007). However, expression of the $\mathrm{G}$ protein is essential for significant replication of BRSV in vivo and mutations (antigenic variation) in the immunodominant region of the $\mathrm{G}$ protein have been described as potentially contributing to the lack of cross-protection seen between vaccine and field isolates (Valarcher and Taylor 2007). The level of homology of the G protein seen between the challenge and more than 30 field strains was above 93\%, instilling confidence on the ability of the vaccine to maintain the level of efficacy seen in this study in the field.

Bovine Viral Diarrhoea Virus (BVDV) is a pestivirus consisting of two genotypes (I and II) and two biotypes (cytopathogenic (CP) and non-cytoptahogenic (NCP)). The BVDV-1 is widely distributed worldwide and represents over $90 \%$ of the BVDV strains in the EU, whereas BVDV-2 is mainly found in North America with a relatively low prevalence in the EU (Lindberg et al. 2006). Pestiviruses have demonstrated a high degree of sequence conservation (Don ofrio et al. 2006), however, some reports describe significant antigenic diversity (Lindberg et al. 2006) with Fulton et al. (2003) suggesting that the high antigenic diversity associated with BVDV genotypes imposes a critical constraint on the development of efficacious vaccines to control the disease.

Glycoprotein E2 is described as the immunodominant glycoprotein inducing the production of neutralizing antibodies after natural infection or following immunization (Donofrio et al. 2006) and the most immunogenic protein of BVDV, eliciting high titres of neutralizing antibodies after infection (Donis et al. 1988). Whilst E2 epitopes were found generally to be highly conserved among BVDV isolates, when represented by the monoclonal antibodies, relatively weak cross-reactions were seen associated with variation in this antigen (Deregt et al. 1998). The close proximity with respect to the E2 epitope between the challenge strain used and the published BVDV field strains provides further reassurance on the relevance of the data seen in this study for application in the field. 
Finally, unlike the situation with the key BRD viruses, Mannheimia haemolytica is a commensal organism of the respiratory tract thereby ensuring that the immunology associated with this pathogen is complicated. Whilst there are numerous serotypes of $M$. haemolytica, the two that are associated with the vast majority of clinical cases are the A1 and A6 serotypes. Apart from the nature of their capsules, serotypes A1 and A6 are considered extremely similar (Rice et al. 2007). Moreover, cross-protection with the A6 serotype is widely described in the literature due to the antigenic closeness of A1 and A6 and further confirmed by a phylogenic analysis that indicated over $98 \%$ of gene sequence similarity between A1 and A6 (Magnier 2014).

In summary, the analysis conducted in this study on the viruses and bacteria focused on the key antigens for each of them, comparing the challenge strains to an array of global and European strains, including many recent strains with known sequences. The results obtained support that there is sufficient genetic proximity between the challenge strains used and the field strains compared for there to be a high likelihood of cross-protection being afforded by the vaccine studied and for the efficacy data to be relevant for field situations.

It is clear that for at least some of the key antigens causing BRD there is a definite potential for antigenic diversity within field strains to reduce the level of protection possible from vaccination approaches. This increases the need for on-going monitoring of the level of antigenic diversity within field strains and the suitability of existing vaccine antigens being used. One way to address this is to use challenge strains in efficacy studies that are representative of the diversity that exists in the field. This study has shown that for the Bovalto Respi range there exists a strong efficacy profile over 6 months against viral strains that are representative of current strains existing in the field.

\section{Acknowledgement}

Challenge strains for the Pi3, BRSV, BVD experimental infections were kindly provided by Kamil Kovařčík, Veterinary Research Institute Brno, Czech Republic.

\section{References}

Ames Trevor R 2007: Shipping Fever and Calf Pneumonia in Cattle. Presentation for Department of Veterinary Population Medicine College of Veterinary Medicine University of Minnesota. Available at: http://www.aawv. net/MembersOnly/Ames\%20Presentation.pdf. Last modified May 15, 2007. Accessed September 26, 2016

Bednarek D, Szymańska-Czerwińska M, Dudek K 2012: Bovine Respiratory Syndrome (BRD) Etiopathogenesis, Diagnosis and Control. In: A Bird's-Eye View of Veterinary Medicine. Dr. Carlos C. Perez-Marin (Ed.), pp. 363-378. Available at: http://cdn.intechopen.com/pdfs-wm/28682.pdf. Last modified: February 22, 2012. Accessed August 19, 2016

Callan Robert J 2002: Biosecurity and bovine respiratory disease. Vet Clin Food Anim 18: 57-77

Deregt D, Van Rijn PA, Wiens TY, Van den Hurk J 1998: Monoclonal antibodies to the E2 protein of a new genotype (type 2) of bovine viral diarrhea virus define three antigenic domains involved in neutralization. Virus Res 57: 171-181

Donis RO, Corapi W, Dubovi EJ 1988: Neutralizing monoclonal antibodies to bovine viral diarrhoea virus bind to the $56 \mathrm{~K}$ to $58 \mathrm{~K}$ glycoprotein. J Gen Virol 69: 77-86

Donofrio G, Bottarelli E, Sandro C, Flammini CF 2006: Expression of Bovine Viral Diarrhea Virus glycoprotein E2 as a soluble secreted form in a mammalian cell line. Clin Vaccine Immunol 13: 698-701

Ellis JA 2010: Bovine parainfluenza-3 virus. Vet Clin North Am Food Anim Pract 26: 575-593

Ewers C, Lübke-Becker A, Wieler LH 2004: Mannheimia haemolytica and the pathogenesis of enzootic bronchopneumonia. Berl Munch Tierarztl Wochenschr 117: 97-115

Fulton RW, Ridpath JF, Confer AW, Saliki JT, Burge LJ, Payton ME 2003: Bovine viral diarrhoea virus antigenic diversity: impact on disease and vaccination programmes. Biologicals 31: $89-95$

Gentry MJ, Confer AW, Holland SG 1998: Comparison of the toxic and antigenic properties of single bovine isolates of Pasteurella haemolytica representing five serotypes and an untypable strain. Vet Microbiol 16: 351-367

Henrickson KJ 2003: Parainfluenza viruses. Clin Microbiol Rev 16: 242-264

Horwood PF, Horwood PF, Gravel JL, Mahony TJ 2008: Identification of two distinct bovine parainfluenza virus type 3 genotypes. J Gen Virol 89: 1643-1648 
Lindberg A, Brownlie J, Gunn GJ, Houe H, Moennig V, Saatkamp HW, Sandvik T, Valle PS. 2006: The control of bovine viral diarrhoea virus in Europe: today and in the future. Rev sci tech Off int Epiz 25: 961-979

Magnier S 2014: The impact of early calfhood disease. Veterinary Ireland Journal 4: 267-269

Meyer G, Deplanche M, Schelcher F 2008: Human and bovine respiratory syncytial virus vaccine research and development. Comp Immunol Microbiol Infect Dis 31:191-225

Monograph Mannheimia vaccine (Inactivated) for Cattle - Ph.Eur. 04/2013:1944

Morton R J, Simons KR, Confer AW 1996: Major outer membrane proteins of Pasteurella haemolytica serovars 1 - 15: comparison of separation techniques and surface-exposed proteins on selected serovars. Vet Microbiol 51: $319-330$

Rice JA, Carrasco-Medina L, Hodgins DC, Shewen PE 2007: Mannheimia haemolytica and bovine respiratory disease. Anim Health Res Rev 8: 117-128

Snowder GD, Van Vleck LD, Cundiff LV, Bennett GL 2006: Bovine respiratory disease in feedlot cattle: Environmental, genetic and economic factors. J Anim Sci 84:1999-2008

Valarcher JF, Hägglund S 2006: Viral respiratory infections in cattle. World Buiatrics Congress. Available at: https://www.researchgate.net/publication/265763919. Last modified: September 18, 2014. Accessed August 19,2016

Valarcher JF, Taylor G 2007: Bovine respiratory syncytial virus infection. Vet Res 38: 153-180

Zechinon L, Fett T, Desmecht D 2005: How Mannheimia haemolytica defeats host defence through a kiss of death mechanism. Vet Res 36: 133-156 